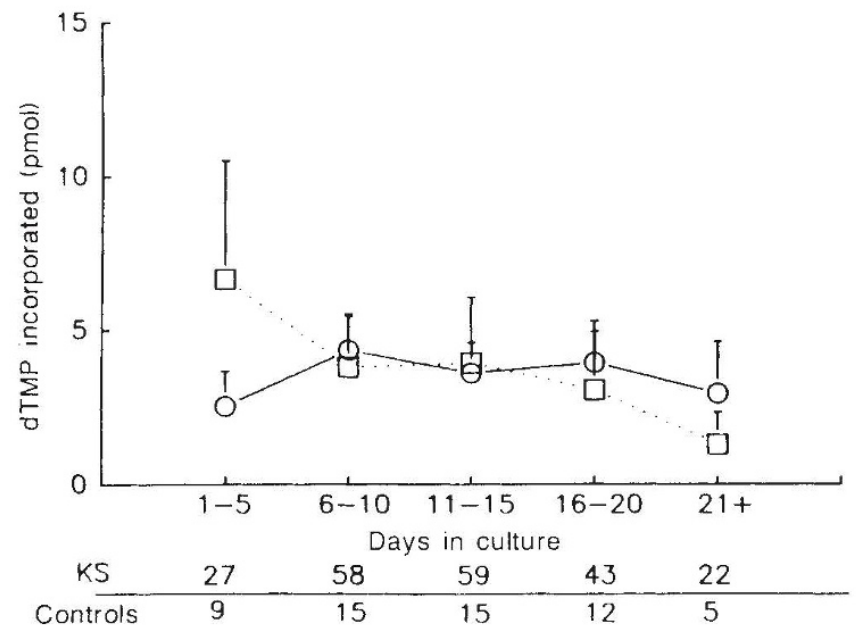

Fig. 3 Particulate polymerase activity in supernatant fluids of lymphocyte cultures. (O), KS patients in convalescent (afebrile) phase; $(\square)$, afebrile controls. The number of cultures tested at each time interval is shown on the horizontal line. Vertical brackets indicate $95 \%$ confidence intervals.

polymerase activity detected in the co-cultures. In one case however, syncytium formation was observed in a co-culture from HPB-ALL. Electron microscopic examination of this culture showed viral particles, subsequently identified as measles virus by specific immunofluorescence and radioimmunoprecipitation assays. The patient had been vaccinated with measles-mumpsrubella trivalent vaccine 8 days before onset of disease. It was concluded that the agent was measles vaccine virus.
Using standard methods for reverse transcriptase detection, we have been unable to demonstrate a retroviral aetiology for $\mathrm{KS}$. The level and pattern of DNA polymerase activity detected in $\mathrm{KS}$ patients in our study were similar to those previously reported ${ }^{2}$. In contrast to their study, cultures from our controls had values and patterns of activity similar to cultures from our $\mathrm{KS}$ patients. In addition, the low-level DNA polymerase activity detected in our cultures showed a template:primer preference consistent with cellular DNA polymerase and inconsistent with retroviral RNA-dependent DNA polymerase. Moreover, we have been unable to detect reverse transcriptase activity in repeated co-cultures of KS patients' lymphocytes with numerous human cell lines. Our experience with recovery of measles virus and its demonstration within cultured lymphocytes by electron microscopy emphasizes the danger in assuming that agents recovered from patients with $\mathrm{KS}$ may have a causative role.

Therefore, we believe that the aetiology of KS remains unknown. If a retrovirus is involved in the causation or the pathogenesis of this disease, techniques more sensitive than the assays presently available will be required to establish its presence.

We thank Drs Sandra Chang and Karen Yamaga for discussion and technical advice, Faith Caplan, Donna Ching, Terry Rota and Caroline Fong for technical support. Supported in part by the Hawaii Heart Association and by the NIH.

Received 12 September; accepted 11 November 1988

1. Kawasaki, T. Jap. J. Allerg. 16, 178-222 (1967)

2. Burns, J. C. et al. Nature 323, 814-816 (1986).

3. Shulman, S. T. Rowley, A. Lancet ii, 545-546 (1986).

4. Ho, D. D., Kaplan, J. C., Rackauskas, I. E. \& Gurney, M. E. Science 239, 1021-1023 (1988)

5. Rho, H. M., Poiesz, B., Ruscetti, F. W. \& Gatlo, R. C. Virology 112, 355-360 (1981).

6. Melish, M. E., Hicks, R. M. \& Larson, E. J. Am. J. Dis. Child. 130, 599-607 (1976).

7. Hoffman, A. D., Banapour, B. \& Levy, J. A. Virology 147, 326-335 (1985).

\title{
Erratum: Drosophila homoeotic genes encode transcriptional activators similar to mammalian OTF-2
}

\section{Markus Thali, Michael M. Müller, Mauro DeLorenzi, Patrick Matthias \& Mariann Bienz}

Nature 336, 598-601 (1988)

a

pCA

pCU

pCO

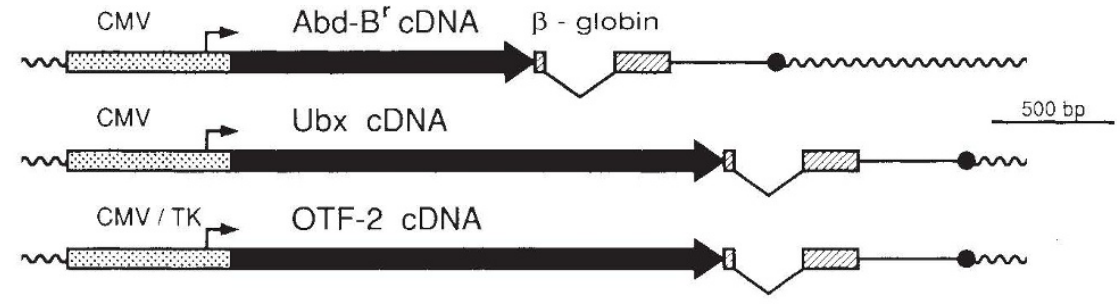

$b$

OVEC

ov

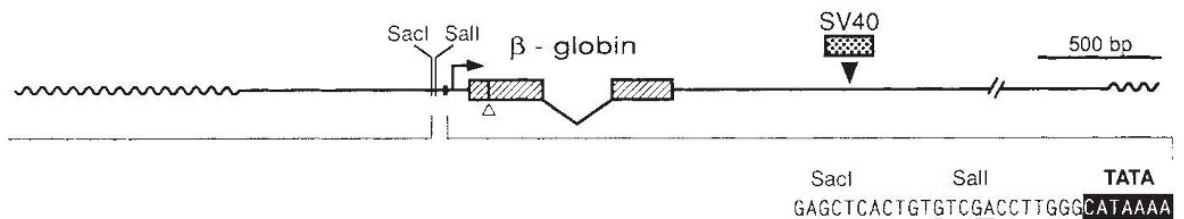

B

GAGCTCCACCCGCGCGCTTTGTITTATIAICCACAIYATCAGCGgCATTATTGITATTATTGGCCCTCATACCGTCGACCTTGGGCATAAAA

E

Octa1

Octa2

Bsub

4GRE

2SP1
GAGCTCGAGTCAATTAAATGTCAATTAAATGCGTCAGCGTCGACCTTGGGCATAAAA

GAGCTCGAGCCCGGGGTAATTTGCATTT TCTACTAGTCGACCTTGGGCATAAAA

GAGCTCTTATTTGCATTTTGTCATAAAA
IN this biological letter, an unrevised version of Fig. 1 was printed. The correct version is shown here. 